\title{
Digital Rise-Time Discrimination of Large-Area Avalanche Photodiode Signals in X-Ray Detection
}

\author{
L. M. P. Fernandes, P. C. P. S. Simões, J. M. F. dos Santos, and R. E. Morgado
}

\begin{abstract}
The response of a large-area avalanche photodiode to $\mathrm{X}$-rays was investigated by applying pulse-shape discrimination techniques based on rise time. The method employed analog preshaping with time constants of $200 \mathrm{~ns}$ followed by digital signal processing in a commercial 125-MHz digitizer. Pulse rise-time discrimination was applied to improve detector energy resolution, background level, and peak distortion. Electronic noise pulses can be efficiently removed at the expense of data-acquisition throughput.
\end{abstract}

Index Terms-Avalanche photodiode, pulse-discrimination efficiency, rise-time discrimination, $\mathrm{X}$-ray detection.

\section{INTRODUCTION}

I NTEREST in the use of avalanche photodiodes (APDs) has increased recently with the appearance of commercially available large-area APDs that deliver higher charge gains at lower bias voltages, with improved spatial uniformity. Windowless large-area APDs with extended sensitivity in the ultraviolet and soft X-ray regions are also commercially available.

Large-area APDs are used mainly as optical photosensors coupled to scintillators for $\mathrm{X}$ - and $\gamma$-ray detection, as a replacement for photomultiplier tubes [1]-[4]. Applications include instrumentation of the large electromagnetic calorimeter of the CERN CMS detector for high-energy physics [1], [5], [6] and the photosensor readout of crystals and plastic scintillators for $\gamma$-ray spectrometry in nuclear physics [7], [8] and for positron emission tomography instrumentation in medicine [9], [10]. Although their use as X-ray spectrometers in the $0.5-20 \mathrm{keV}$ range has been suggested [11]-[13], low-energy X-ray detection techniques with APDs were mainly developed to measure chargecarrier properties of the device, using X-rays as a reference for light measurements [1], [13], [14].

The application of large-area APDs (LAAPDs) to energy-dispersive X-ray fluorescence (EDXRF) analysis has been investigated, and energy resolutions similar to those of proportional counters have been achieved [15]. Additionally, the use of LAAPDs in an experiment to measure the Lamb shift of the

Manuscript received November 23, 2001; revised April 5, 2002. This work was supported by Project FIS/13140/98-13140. The work of R. E. Morgado was supported by the Gulbenkian Foundation and the Foundation for Luso-American Development (FLAD).

L. M. P. Fernandes, P. C. P. S. Simões, and J. M. F. dos Santos are with the Physics Department, University of Coimbra, P-3004-516 Coimbra, Portugal (e-mail: jmf@gian.fis.uc.pt).

R. E. Morgado is with Los Alamos National Laboratory, Los Alamos, NM 87545 USA.

Digital Object Identifier 10.1109/TNS.2002.801518
$2 S-2 P$ atomic states in muonic hydrogen $(\mu \mathrm{p})$ is being considered [16]. The experiment, to be carried out at the Paul Scherrer Institute (PSI), Switzerland, in collaboration with nine institutions, consists in obtaining long-lived $\mu \mathrm{p}$ atoms in the $2 S$-metastable state by stopping a low-energy $\mu^{-}$beam in a small volume of low-pressure hydrogen in a 5-T magnetic field. A pulsed beam from a tunable laser induces the $2 S-2 P$ transition in $\mu \mathrm{p}$, and the $1.9-\mathrm{keV} \mathrm{X}$-ray photons resulting from the $2 P-1 S$ deexcitation will be detected. Measuring the coincidences between the laser pulse and the $1.9-\mathrm{keV} \mathrm{X}$-rays as a function of the laser wavelength, the Lamb shift can be determined. Low counting rates are expected, and the $1.9-\mathrm{keV}$ $\mathrm{X}$-ray background will be reduced by gating its coincidence with the signal resulting from the high-energy electron produced in the subsequent muon decay.

However, improvements on the performance of LAAPDs for $\mathrm{X}$-ray detection, mainly in the low-energy range, are needed. One way to improve this performance may be through the application of pulse discrimination techniques. Fully developed $\mathrm{X}$-ray pulses can be effectively distinguished from noise, distorted X-ray pulses, and pulses resulting from charged-particle interactions.

Digital signal-processing techniques and, in particular, digital rise-time discrimination have been proved to be a simple but effective method to improve detector performance through peak enhancement and background suppression [17]-[20]. In this paper, we investigate the application of digital signal-processing techniques to LAAPDs to improve their response to $\mathrm{X}$-rays by means of pulse rise-time discrimination.

\section{RATIONALE}

APDs are compact monolithic devices made of a silicon $\mathrm{p}-\mathrm{n}$ junction where the internal electric field can reach high enough intensities to produce electron multiplication by impact ionization [1], [21], [22]. When high voltage is applied to the APD, only a small region of the p-layer in the front part of the diode remains undepleted-(I) the drift region, Fig. 1. The electric field in this region is low, but in (II), the depleted region, it increases with depth, reaching a maximum near the $\mathrm{p}-\mathrm{n}$ junction. An incident photon absorbed in either the drift region or the p-depleted layer produces electron-hole pairs. The resulting electrons are accelerated toward the $\mathrm{n}^{+}$-contact, undergoing avalanche multiplication in the high electric field near the junction. Charge gains of a few hundred are typical and vary exponentially with applied voltage. 


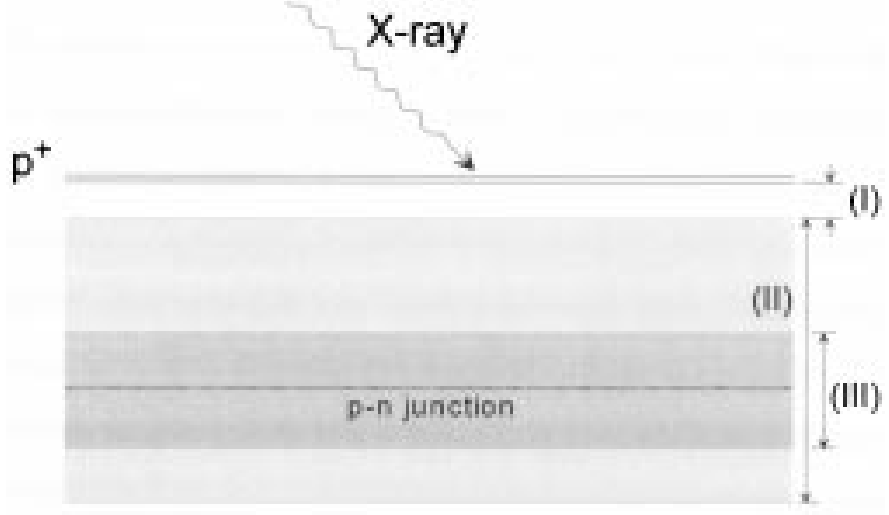

$\mathrm{n}^{+}$

Fig. 1. Schematic diagram of typical avalanche photodiode section: (I) drift region; (II) depleted region; (III) multiplication region.

For the LAAPD used in this paper, ${ }^{1}$ the thickness of the drift region is about $10 \mu \mathrm{m}$, while the charge-multiplication region is about $20-\mu \mathrm{m}$ deep into the depleted region and has a thickness of about $20 \mu \mathrm{m}$ [21]. Thus, the detection efficiency decreases rapidly for $\mathrm{X}$-ray energies above $5 \mathrm{keV}$, and a significant number of X-rays interact deep into the multiplication region.

Fig. 2 depicts a pulse-height distribution measured with the LAAPD for 5.9-keV X-rays. The distribution deviates from a Gaussian due to a low-energy tail. This tail results from two possible sources: 1) X-ray absorptions that occur deep in the photodiode, within the avalanche multiplication region (III), undergo only partial amplification, and 2) X-rays that interact in the reduced electric field, in the undepleted p-region, lose primary charge carriers to either the $\mathrm{p}^{+}$-contact or to traps. Both types of events result in smaller amplitude pulses. For the former type, amplitudes should decrease continuously to zero. The electronic-noise tail in the low-energy region limits the lowest detectable $\mathrm{X}$-ray energy.

$\mathrm{X}$-ray absorption in the undepleted p-region gives rise to pulses with long time-responses because traps may hold electrons for tens to thousands of nanoseconds [21]. The time response for events that occur in the multiplication region is faster than for fully amplified events. These different time responses may result in measurable pulse rise-time differences, allowing for discrimination and rejection of such anomalous pulses and, consequently, for improved detector performance.

\section{EXPERIMENTAL SETUP}

The LAAPD was installed inside a light-tight box to shield it from ambient light. Experimental studies were performed using direct irradiation of the detector with ${ }^{5 \check{5}} \mathrm{Fe} \mathrm{X}$-ray source or X-ray fluorescence from samples positioned in front of the LAAPD in $90^{\circ}$ excitation-detection geometry. To delimit the incident $\mathrm{X}$-ray beam, 2- and 10-mm lead collimators were used for each case, respectively.

LAAPD signals were fed through a low-noise charge preamplifier (Canberra 2004, with a sensitivity of $45 \mathrm{mV} / \mathrm{MeV}$ ) and a spectroscopy amplifier (HP5582A, with gains of 16 to

${ }^{1}$ Advanced Photonix, Inc., Camarillo, CA.

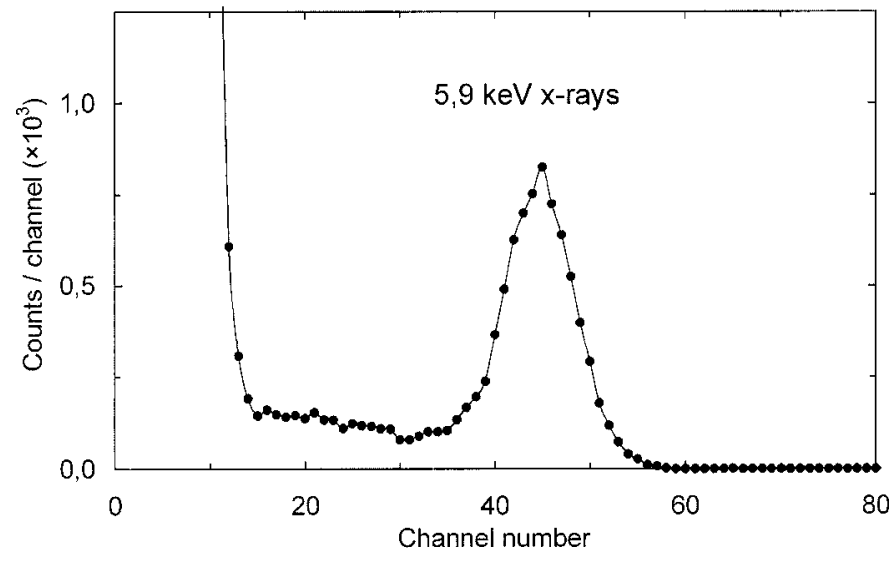

Fig. 2. Typical pulse-height distribution for the LAAPD used in this paper for 5.9-keV X-rays.

64) to a digital pulse-height analyzer (DPHA) for further processing. Integration and differentiation shaping-time constants of $200 \mathrm{~ns}$ were used in the main amplifier for optimum detector performance [13], [15].

The DPHA was composed of a commercially available digitizer PCI board ${ }^{2}$ and the data-processing host computer. The digitizer board has a 12-bit analog-to-digital converter (ADC), and its maximum $125-\mathrm{MHz}$ sampling frequency was used in this work. It has complex triggering capabilities, including databurst acquisition after or before a certain time interval measured from the instant when the trigger threshold is reached. The input-voltage range is $0-1 \mathrm{~V}$, but only $90 \%$ of the ADC full scale is used, as a dc offset is added to the signal prior to digitization. The trigger threshold is set just above the characteristic photodiode electronic noise level for the chosen amplifier gain to avoid dead-time losses analyzing noise pulses.

Pulse-height analysis is performed with a peak-search algorithm, an eighth-order polynomial fitting algorithm, and a modified Newton-tangent method [23]-[25] to determine the peak maximum. If the pulse amplitude is outside of a predefined range, or if more than six iterations are needed for the Newton method to converge, the pulses are rejected. Pulse rise time is defined as the time required for the pulse to increase from 10 to $90 \%$ of its maximum amplitude. The different algorithms have been developed for former applications [25] and fine-tuned for this LAAPD X-ray detector-e.g., the eighth-order polynomial fitting and the maximum number of iterations allowed, before convergence is achieved, were chosen for the best pulse discrimination performance and/or for good compromise between pulse discrimination performance and processing time.

The pulse-height and the rise-time distributions are stored simultaneously. The pulse-height distribution is divided into a series of partial distributions on the basis of rise time. The rise-time range of each partial distribution is $8 \mathrm{~ns}$, limited only by the digitizer sampling frequency. For pulse-amplitude, peak-area, and energy-resolution measurements, the pulse-height distributions were fitted to a Gaussian function superimposed on a linear background from which the centroid, the area, and the full-width at half-maximum were determined. The peak-to-background ratio was defined as the Gaussian area

${ }^{2}$ PDA12A-1997, Signatec, Corona, CA. 


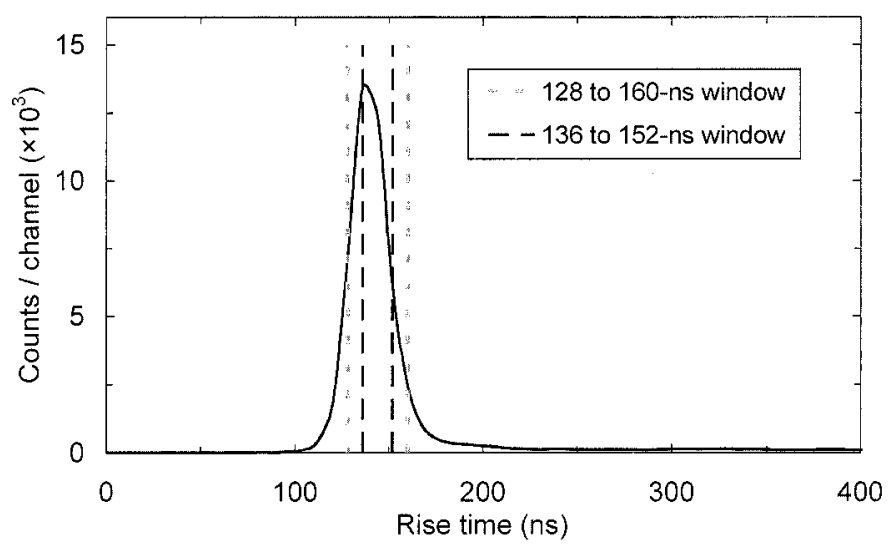

Fig. 3. Pulse rise-time distributions for 5.9-keV X-rays using 200-ns shaping time constants.

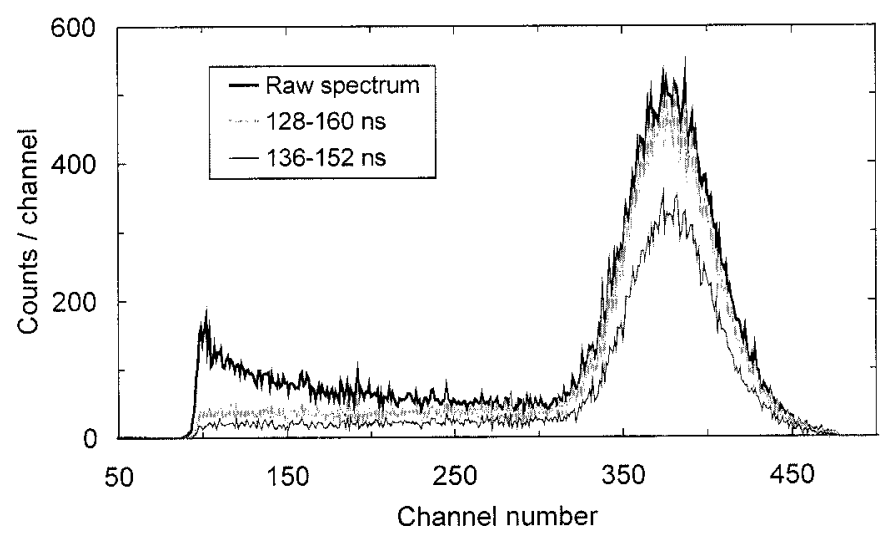

Fig. 4. Pulse-height distributions of different rise-time windows together with the raw spectrum for $5.9-\mathrm{keV} \mathrm{X}$-rays.

divided by the area under the fitted linear background for the peak region.

\section{EXPERIMENTAL RESULTS AND DISCUSSION}

In Fig. 3, we present the pulse rise-time distributions of the 5.9-keV X-rays detected by a 16-mm-diameter LAAPD for the conditions described above. The dashed lines delimit two time windows of 128-160 and 136-152 ns.

Fig. 4 depicts the pulse-height distributions corresponding to those time windows together with the raw spectrum (total pulse-height distribution without any discrimination). For each partial spectrum, energy resolution and signal-to-background ratio show small improvements from $16.5 \%$ and 23 (raw spectrum) to $16.1 \%$ and 27 or $15.4 \%$ and 29 , respectively. However, the relevant feature is the significant reduction of the low-energy tails. This is an important advantage when weaker low-energy lines are superimposed on the tail. For these analyses, rise-time discrimination will result in a significant improvement in the detection sensitivity due to background reduction.

In Fig. 5, we present the raw distribution and three partial pulse-height distributions for time windows outside and inside the 128- to 160-ns rise-time interval. It is observed that a significant number of events with both short and long rise times correspond to events with lower amplitudes than those within the time window. In Table I, the features of the raw spectrum and the relevant partial spectra are characterized according to peak

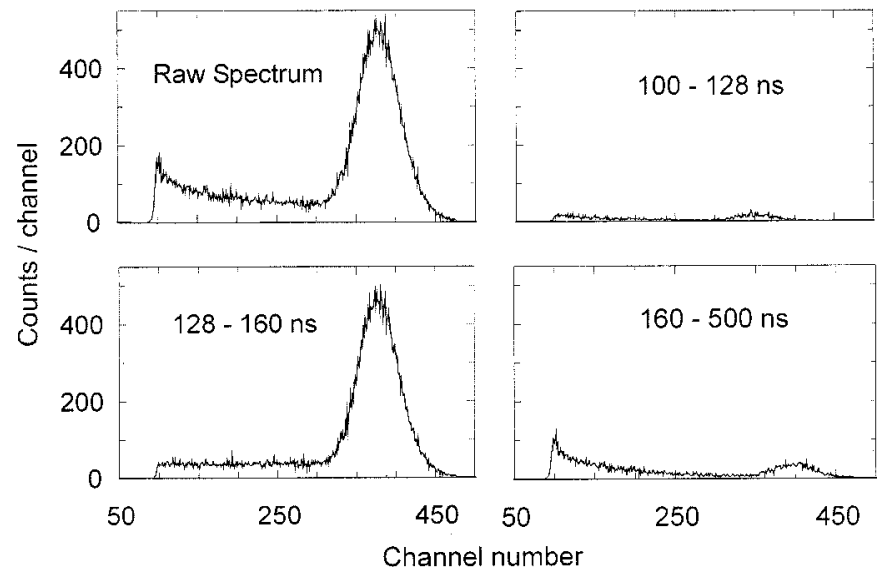

Fig. 5. Raw spectrum and partial spectra for time windows outside and inside the 128 - to 160 -ns rise-time interval.

TABLE I

Parameters of the RaW SPECTRUM and the ReleVant Partial SPeCtra OBTAINED WITH DPHA FOR 5.9-keV X-RAYS

\begin{tabular}{ccccc}
\hline $\begin{array}{c}\text { Rise time } \\
\text { window }\end{array}$ & Centroid & $\begin{array}{c}\text { Energy } \\
\text { resolution }\end{array}$ & $\begin{array}{c}\text { Peak-to- } \\
\text { Background }\end{array}$ & Relative Area \\
\hline Total range & 379.1 & $16.5 \%$ & 23.4 & $100 \%$ \\
$128-136 \mathrm{~ns}$ & 365.3 & $15.2 \%$ & 38.5 & $16 \%$ \\
$136-144 \mathrm{~ns}$ & 375.6 & $15.3 \%$ & 29.1 & $32 \%$ \\
$144-152 \mathrm{~ns}$ & 383.6 & $14.9 \%$ & 31.7 & $30 \%$ \\
$152-160 \mathrm{~ns}$ & 389.8 & $14.2 \%$ & 24.3 & $12 \%$ \\
\hline
\end{tabular}

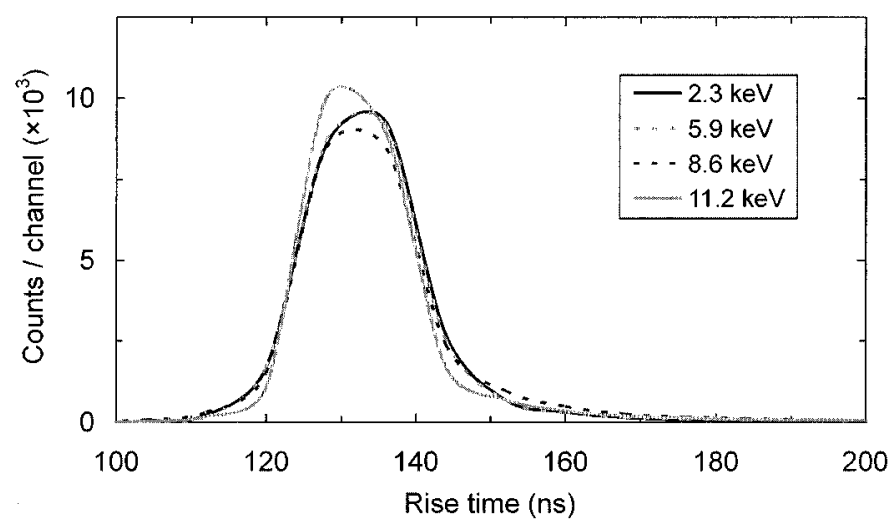

Fig. 6. Pulse rise-time distributions for different X-ray energies using 200-ns shaping time constants.

centroid, energy resolution, peak-to-background ratio, and peak relative area. A slight increase of the peak centroid position of the partial pulse-height distributions with rise time is noticeable due to ballistic deficit. This effect could be corrected after processing the partial spectra, thereby improving the energy resolution and distortions of the final spectrum that result from the sum of several partial spectra. A compromise has to be made between the peak counting efficiency and the pulse-discrimination efficiency. A digital sampling rate higher than $125 \mathrm{MHz}$ would improve the discrimination technique by dividing the partial spectra into narrower rise-time windows.

Fig. 6 depicts several pulse rise-time distributions for different X-ray energies, using the same trigger threshold above 


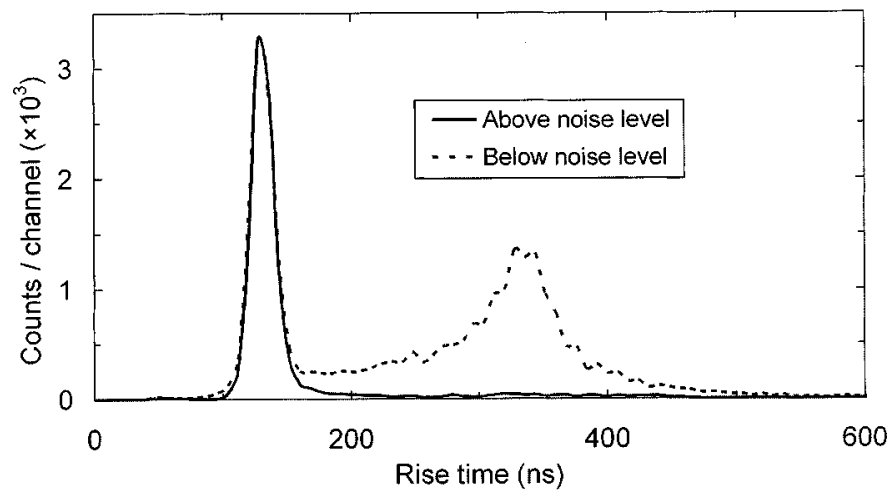

Fig. 7. Pulse rise-time distributions for 5.9-keV X-rays using 200-ns shaping time constants and two different trigger thresholds in the DPHA: just above and below the electronic noise level.

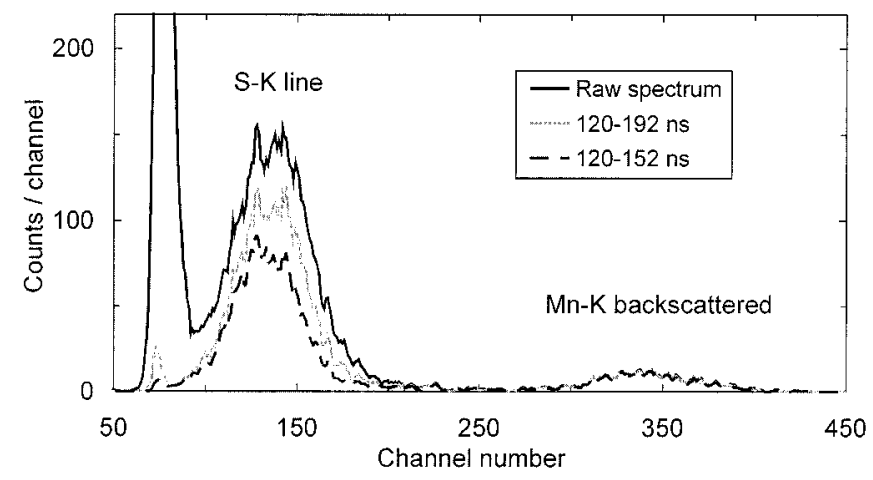

Fig. 8. Pulse-height distributions for the sulphur K-line using 200-ns shaping time constants.

the noise level. The distributions were normalized to the total area. No significant dependence of the rise-time distribution on the X-ray energy is observed.

Pulse rise-time discrimination allows a lowering of the minimum detectable X-ray energy. Fig. 7 depicts two rise-time distributions obtained for 5.9-keV X-rays using different trigger thresholds in the DPHA: just above and below the electric noise level. The figure shows that the electronic noise pulses have longer rise times and, thus, can be efficiently discriminated from the X-ray pulses. However, lowering the trigger threshold in the DPHA results in a reduced data-acquisition throughput due to the time required for analyzing the noise pulses.

We have analyzed the LAAPD response to fluorescence from sulphur and silicon samples excited with a ${ }^{5 \check{5}} \mathrm{Fe} \mathrm{X}$-ray source. In Fig. 8, we present the sulphur total spectrum and two partial spectra obtained for rise-time discrimination windows of 120-192 and 120-152 ns. The energy resolution and the peak-to-background ratio have improved from $32.1 \%$ and 8.6 to $31.9 \%$ and 53 ( $81 \%$ efficiency) or $30.6 \%$ and 51 (57\% efficiency), respectively.

The total spectrum and several different partial spectra are depicted in Fig. 9, spanning the full rise-time range. Table II details the features of the total spectrum and the relevant partial spectra for the sulphur K-line. It is observed that ballistic deficit correction could improve the detector energy resolution to values below $29 \%$ with an acquisition efficiency of about $90 \%$.

In Fig. 10, the silicon raw spectrum and two partial spectra obtained for rise-time discrimination windows of 120-192

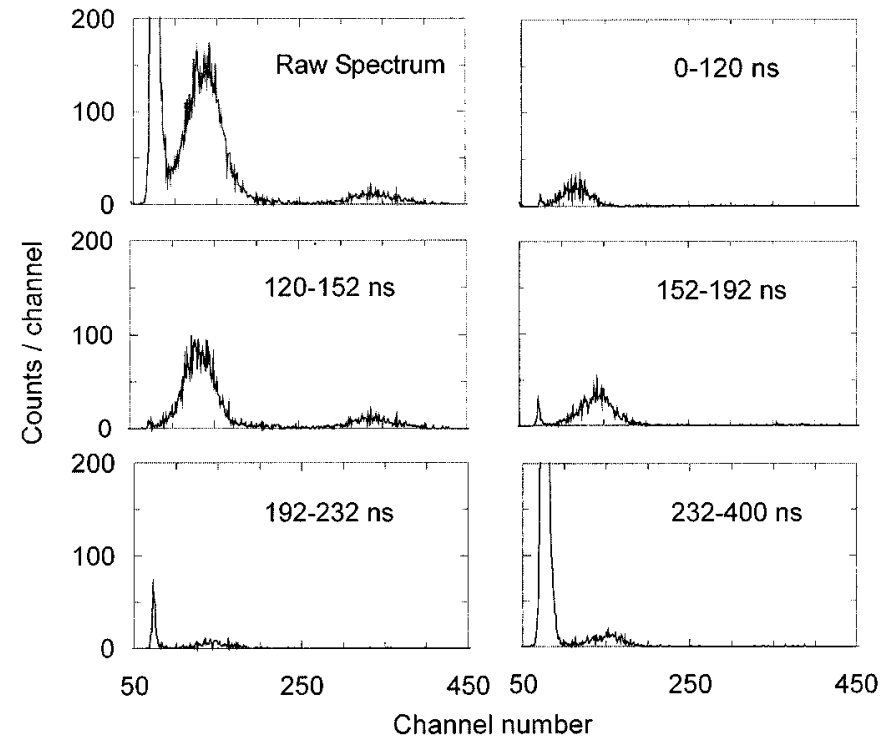

Fig. 9. Raw spectrum and pulse-height distributions of different rise-time windows for sulphur fluorescence.

TABLE II

Parameters of the RaW SPeCtrum and the Relevant Partial SPectra OBTAINED WiTH DPHA FOR THE SULPHUR K-LINE

\begin{tabular}{ccccc}
\hline $\begin{array}{c}\text { Rise time } \\
\text { window }\end{array}$ & Centroid & $\begin{array}{c}\text { Energy } \\
\text { resolution }\end{array}$ & $\begin{array}{c}\text { Peak-to- } \\
\text { Background }\end{array}$ & Relative Area \\
\hline $\begin{array}{c}\text { Total range } \\
80-120 \mathrm{~ns}\end{array}$ & 136.4 & $32.1 \%$ & 8.5 & $100 \%$ \\
$120-136 \mathrm{~ns}$ & 127.6 & $28.0 \%$ & 45.0 & $11 \%$ \\
$136-152 \mathrm{~ns}$ & 136.0 & $28.1 \%$ & 110 & $27 \%$ \\
$152-192 \mathrm{~ns}$ & 142.1 & $28.2 \%$ & 30.5 & $21 \%$ \\
\hline
\end{tabular}

and 120-152 ns are depicted. For the raw spectrum, the noise behavior on the peak region is complex and not well known and, thus, the areas corresponding to the $\mathrm{K}-\mathrm{Si}$ peak and the respective background cannot be determined with a reasonable precision. The energy resolution and the peak-to-background ratio of the silicon K-line achieve $38 \%$ and 4.6 or $36 \%$ and 6.1 , respectively. The calcium line on the pulse-height distribution is due to the plasticine used to hold the ${ }^{5 \tilde{5}} \mathrm{Fe}$ source. Table III details the features of the relevant partial spectra for the silicon K-line. Fig. 11 depicts the total spectrum and several relevant partial spectra.

\section{CONCLUSIONS}

We have shown that the X-ray response of large-area avalanche photodiodes can be improved by pulse rise-time discrimination. Both measured energy resolution and peak-to-background ratio show some improvements. Low-energy tails are reduced, decreasing the overall background level in the pulse-height distributions. Electronic noise can be efficiently discriminated, but reducing the trigger threshold in the DPHA below the noise level results in longer times for the analysis of noise pulses and reduces the data-acquisition throughput. The discrimination efficiency improves by narrowing the rise-time discrimination windows. However, 


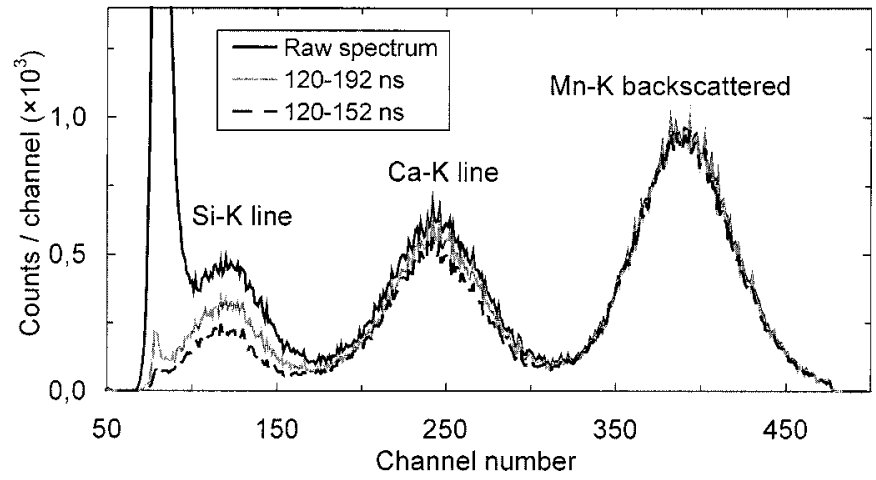

Fig. 10. Pulse-height distributions for the silicon and calcium K-lines using 200-ns shaping time constants.

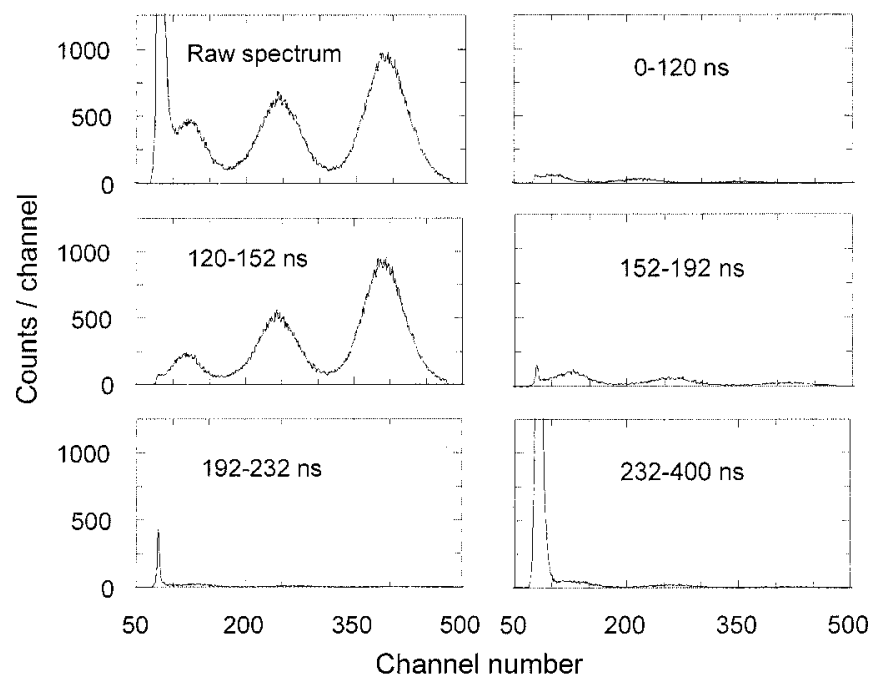

Fig. 11. Raw spectrum and pulse-height distributions of different rise-time windows for silicon fluorescence.

TABLE III

Parameters of the Relevant Partial Spectra ObTained With DPHA FOR THE SILICON K-LINE

\begin{tabular}{ccccc}
\hline $\begin{array}{c}\text { Rise time } \\
\text { window }\end{array}$ & Centroid & $\begin{array}{c}\text { Energy } \\
\text { resolution }\end{array}$ & $\begin{array}{c}\text { Peak-to- } \\
\text { Background }\end{array}$ & Peak Area \\
\hline $80-120 \mathrm{~ns}$ & 103.8 & $29.0 \%$ & 1.59 & 1200 \\
$120-136 \mathrm{~ns}$ & 114.0 & $34.0 \%$ & 3.93 & 3650 \\
$136-152 \mathrm{~ns}$ & 121.3 & $29.6 \%$ & 3.61 & 3440 \\
$152-192 \mathrm{~ns}$ & 126.3 & $33.9 \%$ & 3.14 & 3380 \\
\hline
\end{tabular}

a compromise has to be made between the peak counting efficiency and the pulse-discrimination efficiency.

Future work will include the information contained in the midpoint of each pulse for improvement of pulse-discrimination efficiency.

\section{REFERENCES}

[1] J. P. Pansart, "Avalanche photodiodes for particle detection," Nucl. Instrum. Meth., vol. A 387, pp. 186-193, 1997.
[2] R. Farrell, F. Olschner, E. Frederick, L. McConchie, K. Vanderpuye, M. R. Squillante, and G. Entine, "Large-area silicon avalanche photodiodes for scintillation detectors," Nucl. Instrum. Meth., vol. A 288, pp. $137-139,1990$.

[3] M. Moszynski, M. Kapusta, J. Zalispka, M. Balcerzyk, D. Wolski, M. Szawlowski, and W. Klamra, "Low energy $\gamma$-rays scintillation detection with large area avalanche photodiodes," IEEE Trans. Nucl. Sci., vol. 46, pp. 880-885, 1999.

[4] J. A. M. Lopes, J. M. F. dos Santos, and C. A. N. Conde, "A large area avalanche photodiode as a VUV photosensor readout for gas proportional scintillation counters," Nucl. Instrum. Meth., vol. A 454, pp. $421-425,2000$.

[5] A. Karar, Y. Musienko, and J. C. Vanal, "Characterization of avalanche photodiodes for calorimetric applications," Nucl. Instrum. Meth., vol. A 428, pp. 413-431, 1999.

[6] K. Deiters, Y. Musienko, S. Nicol, B. Patel, D. Renker, S. Reucroft, R. Rusack, T. Sakhelashvili, J. Swain, and P. Vikas, "Properties of the most recent avalanche photodiodes for the CMS electromagnetic calorimeter," Nucl. Instrum. Meth., vol. A 442, pp. 193-197, 2000.

[7] E. Lorentz, S. Natkaniec, D. Renker, and B. Schwartz, "Fast readout of plastic and crystal scintillators by avalanche photodiodes," Nucl. Instrum. Meth., vol. A 344, pp. 64-72, 1994.

[8] R. Farrel, F. Olschner, K. Shah, and M. R. Squillante, "Advances in semiconductor photodetectors for scintillation," Nucl. Instrum. Meth., vol. A 387, pp. 194-198, 1997.

[9] B. Pichler, G. Böning, E. Lorenz, R. Mirzoyan, W. Pimpl, M. Schwaiger, and S. I. Ziegler, "Studies with a prototype high resolution PET scanner based on LSO-APD modules," IEEE Trans. Nucl. Sci., vol. 45, pp. 1298-1302, 1998.

[10] A. Ruru Chen, A. Fremout, S. Tavernier, P. Bruyndonckx, D. Clément, J.-F. Loude, and C. Morel, "Readout of scintillator light with avalanche photodiodes for positron emission tomography," Nucl. Instrum. Meth., vol. A 433, pp. 637-647, 1999.

[11] R. Farrel, K. Vanderpuye, G. Entine, and M. R. Squillante, "High resolution low energy avalanche photodiode $\mathrm{x}$-ray detectors," IEEE Trans. Nucl. Sci., vol. 38, pp. 144-147, 1991.

[12] A. Ochi, Y. Nishi, and T. Tanimori, "Study of a large area avalanche photodiode as a fast photon and soft x-ray detector," Nucl. Instrum. Meth., vol. A 378, pp. 267-274, 1996.

[13] M. Moszynski, M. Szawlowski, M. Kapusta, M. Balcerzyk, and D. Wolski, "Large avalanche photodiodes in x-rays and scintillation detection," Nucl. Instrum. Meth., vol. A 442, pp. 230-237, 2000.

[14] C. P. Allier, H. Valk, V. R. Bom, R. W. Hollander, and C. W. E. van Eijk, "Comparative study of silicon detectors," IEEE Trans. Nucl. Sci., vol. 45, pp. 576-580, 1998.

[15] L. M. P. Fernandes, J. A. M. Lopes, J. M. F. dos Santos, and C. A. N. Conde, "Application of a large area avalanche photodiode in energy dispersive x-ray fluorescence analysis," X-Ray Spectrom., vol. 30, pp. 164-169, 2001.

[16] F. Kottmann, W. Amir, F. Biraben, C. A. N. Conde, S. Dhawan, and T. W. Hänsch et al., "The muonic hydrogen Lamb shift experiment at PSI," Hyperf. Inter., 2002, to be published.

[17] B. Aspacher and A. C. Rester, "Compton continuum suppression by digital pulse shape analysis-Part II," Nucl. Instrum. Meth., vol. A 338, pp. 516-521, 1994.

[18] S. A. Audet, J. J. Friel, T. P. Gagliardi, R. B. Mott, J. I. Patel, and C. G. Waldman, "High resolution energy dispersive spectroscopy with highpurity germanium detectors and digital pulse processing," in 1994 IEEE Conf. Rec., vol. 1, 1995, pp. 155-159.

[19] P. C. P. S. Simões, J. F. C. A. Veloso, J. M. F. dos Santos, and C. A. N. Conde, "Application of the digital pulse processing technique to gas proportional scintillation counters," IEEE Trans. Nucl. Sci., vol. 44, pp. 521-526, 1997.

[20] P. C. P. S. Simões, J. M. F. dos Santos, and C. A. N. Conde, "Digital risetime discrimination for peak enhancement in gas proportional scintillation counter x-ray fluorescence analysis," X-Ray Spectrom., vol. 26, pp. $182-188,1997$.

[21] A. Q. R. Baron and S. L. Ruby, "Time resolved detection of x-rays using large area avalanche photodiodes," Nucl. Instrum. Meth., vol. A 343, pp. 517-526, 1994.

[22] E. M. Gullikson, E. Gramsch, and M. Szawlowski, "Large-area avalanche photodiodes for the detection of soft x-rays," Appl. Opt., vol. 34, pp. 4662-4668, 1995.

[23] P. R. Bevington, Data Reduction and Error Analysis for the Physical Sciences. New York: McGraw-Hill, 1969, p. 100, 208.

[24] J. B. P. S. Simões, P. C. P. S. Simões, and C. M. B. A. Correia, "Nuclear spectroscopy pulse height analysis based on digital signal processing techniques," IEEE Trans. Nucl. Sci., vol. 42, pp. 700-704, 1995.

[25] P. C. P. S. Simões, J. C. Martins, and C. M. B. A. Correia, "A new digital signal processing technique for applications in nuclear spectroscopy," IEEE Trans. Nucl. Sci., vol. 43, pp. 1804-1809, 1996. 